\title{
延伸护理服务在慢性阻塞性肺疾病患者肺康复训练中 的应用
}

\author{
诸海英 黎瑞红* 夏引芳 韩玉琴 费 红 \\ 上海市奉贤区中心医院，上海 201499
}

\begin{abstract}
摘 要: 目的：探讨延伸护理服务在慢性阻塞性肺疾病稳定期患者肺康复训练中的应用效果。方法：采用随机 分组法将 120 例住院患者按单双号分成对照组和试验组。对照组实施常规护理, 试验组在常规护理基础上予以延伸 护理服务为框架的呼吸康复训练。结果: 呼吸困难评分比较: 组内比较, 干预前、后试验组差异有统计学意义 (试 验组 $P<0.001)$; 但组间比较差异均无统计学意义; 6分钟步行距离比较: 干预前后组内、组间比较差异均有统计 学意义 (试验组 $P<0.001$, 对照组 $P=0.03$, 组间 $P<0.001$ ) ; 生活质量比较: 组内比较时, 试验组干预前后的差 异具有统计学意义 $(P<0.001$,$) 组间比较, 试验组和对照组也具有显著差异 (P<0.001)$ 。结论: 延伸护理服 务较传统呼吸康复训练更有利于改善慢性阻塞性肺疾病患者的呼吸困难程度, 提高运动能力, 最终提高患者生活质 量, 值得临床推广应用。
\end{abstract}

关键词：延伸护理服务；慢性阻塞性肺疾病；肺康复；生活质量

\section{一、前言}

慢性阻塞性肺疾病 (Chronic Obstructive Pulmonary Disease, COPD) 是一种以不完全可逆的气流受限为特点的慢 性呼吸道疾病。具有高患病率、高致残率、高死亡率特点, 我国农村和城市地区以上人群总体患病率 $8.6 \%{ }^{[1]}$ 。因反复 发作影响患者的呼吸、运动、心理等各个方面, 导致生存质量下降, 生活难以自理, 给家庭和社会造成沉重的经济负 担。慢性阻塞性肺疾病全球防治倡议 (globalinitiative for chronic obstructivelungdisease, GOLD) 指出COPD是全球第 四大致死的病因, 推荐肺功能分级 II 级及以上的COPD患者均应接受肺康复治疗 ${ }^{[2]}$ 。肺康复是个长期连续的过程, 但 由于医疗资源紧张, 患者住院日的逐渐缩短。大多患者在经过短暂的疾病急性期住院治疗和康复后, 不得不转到社区 或者家庭。延伸护理是指通过一系列的行动设计, 以确保患者在不同的健康照护场所 (如从医院到家庭) 及同一健康 照护场所 (如医院的不同科室) 受到不同水平的协作性与连续性的照护，使患者回归家庭或社区后仍得到持续随访与 指导, 有效应对患者返家后面临的健康问题。实现了医院一社区一家庭的护理服务模式 ${ }^{[3]}$ 。在现阶段, 国内延续护理 模式依旧处于经验总结与体会阶段, 针对单一的疾病或人群, 并没有一个整体、系统的工作模式, 本研究整合社区护 理的力量, 与我院组合成一个医疗团队, 有效的整合了医院、社区护理和保健资源, 高效地减轻了患者及家属的负 担, 更好的保证了对COPD患者家庭护理的延伸。在2018年1月 2019年1月, 我们将延伸服务应用于提高COPD患者的 肺康复训练能力和生活质量, 取得满意效果。现报告如下。

\section{二、对象与方法}

(一) 研究对象

经医院伦理委员会同意, 患者知情同意, 选取2018年1月 2018年12月在我院呼吸科住院的120例COPD患者作为研 究对象。

1. 纳人标准

（1）符合慢性阻塞性肺疾病诊治指南（2018年）的诊断标准，并由医生诊断为慢性阻塞性肺疾病。

(2) 经住院治疗后, 病情稳定者。

（3）患者意识清楚、能通过文字或语言沟通。

(3) 自愿参加本次研究并且签订知情同意书。

2. 排除标准

（1）有严重心、肝、肾功能障碍或恶性肿瘤的患者。

*通讯作者: 黎瑞红, 1973年11月, 女, 汉族, 就职于上海市奉贤区中心医院, 副主任护师/副教授, 硕士。研究 方向: 慢性病管理、老年护理、护理管理、护理教育。 
（2）具有咳嗽、咳痰、喘息症状的其他疾病, 例如不明原因的慢性咳嗽、支气管哮喘、支气管扩张、肺结核、充 血性心力衰竭等。

3. 失访率和干扰因素

由于搬迁和病情加重, 不适合本研究中度COPD患者纳人标准而失访, 失访率 $9 \%$, 两组患者性别、年龄、文化程 度、病程等因素的干扰比较差异无统计学意义 $(P>0.05)$, 具有可比性。

(二) 方法

对照组出院前 2 天及出院当天给予呼吸康复训练的培训, 包括COPD疾病基础知识、戒烟宣教、有效的咳嗽排痰方 法、平喘药物正确使用、运动训练及呼吸肌在肺复康治疗中的重要性、心理与行为干预治疗等。

指导所有出院的COPD患者能够完成居家呼吸康复训练，有效的咳嗽排痰方法；缩唇呼吸和腹式呼吸、呼吸操。 每次康复训练15 20 min, 1 2次/d, 每次锻炼以自我稍感劳累, 但不出现呼吸困难为宜, 并强调家属督促配合的重要 性。并对其定期进行电话随访。试验组在对照组基础上, 组建专科护理团队对病人进行连续的疾病管理, 患者和主要 照顾者共同参与。延伸护理主要包括以下几方面。

( 1 ) 责任护士完成个体化护理评估, 建立健康资料档案

对病人及其家属开展健康教育讲座, 告知责任护士及护士长、社区护士的联系方式, 接受患者咨询。联络社区护 士, 告知病人的基本情况。

(2) 每月社区护士家庭访视和评估

出院后第3、第6个月，护士长、医生、责任护士和社区护士家庭访视和评估。

(3) 电话督导

在患者出院后进行, 为期 6 个月, 每月电话随访一次, 时间为 15 分钟, 目的是了解患者及家属的反馈, 强化向患 者及家属传授的相关知识和技能。

(4) 短信提醒

在患者出院后进行, 为期 6 个月。研究者每周通过短信方式向患者及家属传递健康信息, 共计 24 周。短信根据指 导的内容进行编写, 内容更加简练。

( 5 ) 健康周记

为期6月。内容包括每周呼吸困难测定; 每周测 6 min行走的距离。目的是让患者及家属了解自己的病情, 将学到 的知识和技能在日常生活中应用。

(三) 观察指标

1. 呼吸困难

改良版的英国医学研究会的呼吸困难量表 (modified Medical Research Council, mMRC) mMRC根据患者出现气短 时的活动程度分为 $0 \sim 4$ 共 5 个等级。 0 级计 1 分, 4 级计 5 分。得分越高说明患者呼吸困难症状越严重。 $\mathrm{mMRC}$ 评分是日常 临床实践中评估COPD患者呼吸健康的常用工具，简单易行，灵敏度高，在国内外研究中广泛应用 ${ }^{[4]}$ 。

2. 运动耐力

$6 \mathrm{~min}$ 步行距离 ( $6 \mathrm{MwD})$ 测验: 用以测定研究对象的运动耐力。在测试中要求研究对象尽可能地在走廊往返快步 走, 记录30 m内行走的距离。6MWD分级标准: 轻度慢阻肺: $6 \mathrm{MWD} \geqslant 350 \mathrm{~m}$; 中度慢阻肺: $250 \sim 349 \mathrm{~m}$; 重度慢阻 肺: 150 249 m; 极重度慢阻肺: $\leqslant 149 \mathrm{~m}$ 。

3. COPD评估测试 ( CAT)

是由圣乔治呼吸问卷 (SGRQ) 设计者Jones于 2009 年研发, 是由患者本人独立完成的一种新型、简易的COPD患 者生活质量测试问卷 ${ }^{[3]}$ 。虽包含 8 个问题, 却涉及症状、活动能力、心理、睡眠、乏力等多个方面的内容, 每个问题的 评分范围为 $0 \sim 5$ 分, 总分 $0-40$ 分, $0 \sim 10$ 分为轻微影响, 11 20分为中度影响, 21 30分为严重影响, 31 40分为非常严重 影响。

(四) 统计学方法

应用统计软件SPSS13.0统计分析, 计数资料采用 $x^{2}$ 检验, 计量资料以均数土标准差 $(\bar{x} \pm s)$ 表示, 两组间比较采 用 $t$ 检验。 $P<0.05$ 为差异有统计学意义。

\section{三、结果}

(一) 两组患者呼吸困难评分比较

组内比较, 干预前、后呼吸困难评分均有降低, 不过只有试验组达到了显著水平 [P ( CAT ) 0.001]; 组间比较, 
两组干预前评分差异无统计学意义, 干预6月后试验组呼吸困难评分明显降低, 两组比较差异有统计学意义 $(P<$ $0.001)$, 详见表 1 。

表1 两组患者干预前后呼吸困难评分结果比较 $\left(\bar{x}_{ \pm s}\right)$

\begin{tabular}{cccccc}
\hline 组别 & 例数 & 干预前 & 干预后 & $t$ 值 & $P$ 值 \\
\hline 试验组 & 56 & $1.59 \pm 0.50$ & $1.09 \pm 0.35$ & 6.55 & $<0.001$ \\
对照组 & 53 & $1.64 \pm 0.48$ & $1.45 \pm 0.50$ & -97 & 0.05 \\
$t$ 值 & & -0.56 & -4.40 & & \\
$P$ 值 & & 0.58 & $<0.001$ & \\
\hline
\end{tabular}

（二）两组患者6分钟步行距离比较

组内比较, 试验组及对照组在干预前后差异均有统计学意义 (试验组 $P<0.001$, 对照组 $P=0.03$ ); 组间比较, 干预6月后实验组6分钟步行距离比对照组增加明显, 有统计学意义 $(P<0.001)$, 详见表2。

表2 两组患者训练前后 6 min步行距离比较 $\left(\bar{x}_{ \pm} s\right)$

\begin{tabular}{cccccc}
\hline 组别 & 例数 & 干预前 & 干预后 & $t$ 值 & $P$ 值 \\
\hline 试验组 & 56 & $211.38 \pm 37.41$ & $243.04 \pm 43.77$ & -3.85 & -2.12 \\
对照组 & 53 & $197.69 \pm 31.02$ & $211.61 \pm 32.45$ & & 0.001 \\
$t$ 值 & & 1.99 & 4.09 & & \\
$P$ 值 & & 0.49 & $<0.001$ & & \\
\hline
\end{tabular}

(三) 两组患者生活质量比较

组内比较, 试验组、对照组生活质量均显著提高, 干预前、后比较差异有统计学意义（试验组 $P<0.001$ ) ; 组间 比较, 试验组干预6月后较对照组生活质量提高明显，与对照组比较差异有统计学意义 $(P<0.001)$, 详见表 3 。

表3 两组患者训练前后生活质量比较 $\left({ }^{-} \pm s\right)$

\begin{tabular}{cccccc}
\hline 组别 & 例数 & 干预前 & 干预后 & $t$ 值 & $P$ 值 \\
\hline 试验组 & 56 & $22.39 \pm 3.61$ & $15.02 \pm 2.91$ & 1.90 & 1.98 \\
对照组 & 53 & $22.94 \pm 3.90$ & $21.49 \pm 3.67$ & -8.19 & 0.05 \\
$t$ 值 & & -0.77 & $<0.001$ & & \\
$P$ 值 & & 0.45 & & \\
\hline
\end{tabular}

\section{四、讨论}

慢性阻塞性肺疾病全球倡议 (GOLD) 中特别强调了COPD稳定期患者进行肺康复治疗的地位, 指出各阶段COPD 患者经肺康复后, 其运动能力和生活质量的提高可以维持 9 个月, 但如果缺乏后续正性强化和干预, 12 个月后这种获 益就开始逐渐下降, 所以需要长期坚持肺康复方案, 实现长期、全程、系统的居家康复管理。延伸护理是由美国老年 病协会 ${ }^{[6]}$ 提出, 是护理人员通过电话随访、家庭访视、健康俱乐部等形式为患者提供从医院到社区的信息延续、护理 服务延续、医护患关系延续的护理模式 ${ }^{[7-8]}$ 。该模式的运用，使医院患者家属之间有了更为稳固良好的关系。

(一) 延伸护理模式为慢阻肺稳定期患者提供了一个可借鉴的方案

本研究由医生与护士共同为患者制定更趋合理、科学、可行的出院计划，做到患者从医院到社区“无缝连接”， 通过访视时的观察、教育、评估、指导等方式, 干预患者的认知和行为, 使患者得到全方位的照顾。让医院的护理工 作得到了延展, 形成了 “医院一社区一家庭” 的服务模式, 实现了将住院护理服务延续到社区一家庭, 使患者在不同 健康场所转移时得到全方面、合作性、协调性、延续性的服务。本研究因为有了社区护理资源的参与, 拓展了护理的 服务领域。

（二）延伸护理模式在慢阻肺稳定期患者中显示出良好的护理成效

1. 改善患者的呼吸困难

COPD患者大多为老年患者、文化水平有限, 接受宣教效果差, 自我护理能力水平较低。出院后患者健康意识 淡薄, 离开医护人员监管后, 缺乏规律的呼吸功能锻炼 ${ }^{[9]}$ 。研究表明延伸护理能够改善老年COPD患者院外的呼吸功 能, 维持院内治疗效果 ${ }^{[10]}$ 。同时, 越来越多的证据表明COPD是一种身心共患性疾病 ${ }^{[11]}, \mathrm{COPD}$ 的呼吸困难与心理状况 有密切关系。延续护理建立了医护人员、患者、照顾者的沟通渠道, 重视三者的协调、合作, 帮助患者建立应对疾病 的能力, 保证康复计划有效实施。 
2. 增加患者的运动耐力

由于应用了延续护理, 医护人员的指导、监督、调动激发了患者进行肺康复的主动性和积极性, 以肺康复的方式 实施全身性运动, 使膈肌的肌力和耐力得到充分锻炼和提升, 持续提高慢阻肺患者的股四头肌力量、最大吸气压力, 提高了患者的运动耐力。

3. 提高患者的生活质量

世界卫生组织 ( WHO ) 指出 ${ }^{[12]}$ 生活质量是指不同文化和价值体系中的个体对他们的生存目标、期望、标准以及 所关心的事情相关的生存状况感受。COPD是一种病程漫长, 呈进行性发展的疾病, 患者反复人院治疗, 经济压力随 之加大, 容易出现自责、焦虑、抑郁等情绪障碍。由此可见, COPD从生理、心理及社会等方面都能使患者生活质量 降低。延伸护理是以患者为中心, 对患者生理、心理、及社会状况进行全面评估之后对患者进行健康照护, 同时护士 在随访过程中监测、评估并调整计划等来提高了患者肺康复的效果。此外, 延伸护理实施者引导患者及其家属参与疾 病管理中来，这能对患者的情感及社会系统起到一定的支持作用，由于患者自觉坚持进行肺康复训练，延缓了患者肺 功能的下降、提升了患者的体力及运动耐力, 从而提高患者的生活质量。已有研究显示家属参与肺康复过程有利于康 复效果的提高。

(三) 研究的局限性

延伸护理团队应该是一个多学科的专业团队，但本研究尚缺药剂师、营养师等，可能对护理效果产生一定影响。 应加强不同卫生保健服务之间的协调与整合。肺康复是一个多学科综合干预过程, 应对康复程序和方案进行质量控 制, 使单中心研究成果能够尽快在专业的中心进行示范推广。

综上所述, 延伸护理可以改善患者的运动耐力、呼吸困难、使患者的生活质量提高, 从而减少医疗支出, 合理分 配医疗资源。值得临床推广应用。

\section{参考文献:}

[1]王辰,迟春花,陈荣昌,杨汀,陈亚红,孙永昌.慢性阻塞性肺疾病基层诊疗指南(2018年)[J].中华全科医师杂志, 2018,17 (11):856-870.

[2]中华人民共和国卫生部.2007中国卫生统计年鉴[M].北京:中国协和医科大学出版社, 2007.

[3]陈亚红, 姚婉贞, 康健, 蔡柏蓄, 周新, 刘政, 陈萍, 孙德俊, 郑劲平, 王国扬, 冯玉麟, 徐永健, 贾燕, 路明, 常春, 王䈗宏, 慢性阻 塞性肺疾病患者治疗状况与自我认知的多中心调查研究 [J].中华结核和呼吸杂志, 2010(10):750-753.

[4] 陈小平, 赵壮, 陈燕, 李陆振. 系统化呼吸训练结合优质护理对非小细胞肺癌患者肺通气功能、 mMRC评分及置管时 间的影响[J].护理实践与研究, 2020,17(14):60-62.

[5]Simon Jan,Marie - Astrid Metten,Anthony Chapron,Solenne Marette,Ange - Marie Robert,Stéphanie Guillot,Carole Mailloux,Stéphane Jouneau,Jean - François Viel. Use of the COPD Assessment Test (CAT) to screen for COPD in dairy farmers: AIRBAg study[J]. The Clinical Respiratory Journal, 2020,14(9).

[6]Goligher EC,Laghi F,Detsky ME,et al.Measuring diaphragm thickness With ultrasound in mechanically ventilated patients:feasibility, Reprouducibility and validity.Intensive Care Med, 2015,41(4):73.

[7]陆晨,沈国娣, 邱国琴, 钟紫外. 体质量管理联合延续护理对肥胖相关性阻塞性睡眠呼吸暂停低通气综合征患者正压 通气的影响[J].中国实用护理杂志, 2015,31(05):338-341.

[8]周剑英, 庄翠芳, 张晓燕, 吴水, 许晖. 延续护理对腹膜透析患者遵医行为及相关并发症的影响 [J].中华现代护理杂志, 2016,22(17):2469-2472.

[9]黄渘华.延续护理对COPD伴呼吸衰竭患者自我管理能力的影响[J].当代护士, 2018,25(24):58-60.

[10]许玉红.延续性护理对老年COPD患者院外呼吸功能及生活质量的影响[J].临床护理杂志, 2017,16(4):54-56.

[11]姚海燕,盛文化, 吴大伟.慢性阻塞性肺疾病患者心理分析及伦理学治疗方法[J]. 中国医学伦理学, 2007,20(1):112--113.

[12]Skevington S M,Sartorius N,Amir M.Developing methods for assessing Quslity of life in different cultural settings. The history of the WHOQOL struments.Social Psychiatry and Psychiatric Epidemiology.[J].Copd-journal of chronic Obstructive Pulmonar Disease, 2012,9(9):12-15. 